\title{
Avaliação da Variabilidade de Intervenções Baseadas no Cateter de Artéria Pulmonar. Experiência Brasileira*
}

\author{
Variability in Interventions with Pulmonary \\ Artery Catheter Data. Brazilian Experience
}

Ciro Leite Mendes ${ }^{1}$, Ederlon Rezende ${ }^{2}$, Fernando Suparregui Dias ${ }^{3}$, Álvaro Réa-Neto ${ }^{4}$

\section{RESUMO}

JUSTIFICATIVA E OBJETIVOS: A utilização do cateter de artéria pulmonar (CAP) é ainda fonte de debates, devido aos questionamentos sobre sua segurança e eficácia. Este estudo reproduz, entre uma amostra de médicos brasileiros, outra pesquisa, na qual foi evidenciada a heterogeneidade de condutas guiadas através dos dados fornecidos pelo CAP entre médicos norte-americanos.

MÉTODO: Durante o Congresso Brasileiro de Medicina Intensiva (Curitiba, 2004), foram distribuídos formulários nos quais constavam três casos com dados de CAP e, na metade deles, de ecocardiografia. Foi solicitado aos médicos que assinalassem uma entre seis opções terapêuticas. Determinou-se que uma resposta homogênea resultaria em uma escolha selecionada por pelo menos $80 \%$ dos respondedores.

1. Coordenador da UTI adulto do Hospital de Emergência e Trauma Senador Humberto Lucena e Hospital Santa Paula; Médico Coordenador da UTI Adulto do Hospital Universitário da UFPB, Campus I; João Pessoa, PB;

2. Diretor do Serviço de Terapia Intensiva do Hospital do Servidor Público Estadual Francisco Morato de Oliveira; São Paulo, SP.

3. Chefe da UTI geral do Hospital São Lucas da PUC de Porto Alegre; Professor da faculdade de Medicina da Pontifícia Universidade Católica de Porto Alegre; Porto Alegre, RS.

4. Chefe da UTI adulto do Hospital de Clínicas da Universidade do Paraná; Professor do Departamento de Clínica Médica da Universidade Federal do Paraná; Curitiba, PR.

*Recebido da UTI Adulto do Hospital Universitário Lauro Wanderley, Universidade Federal da Paraíba (UFPB), João Pessoa, PB

Apresentado em 30 de novembro de 2005

Aceito para publicação em 24 de maio de 2006

Endereço para correspondência:

Dr. Ciro Leite Mendes

UTI Adulto - Hospital Universitário Lauro Wanderley

Rua Antônio Lira, 823/101

58045-030 João Pessoa, PB

E-mail: cirolm@uol.com.br

(C)Associação de Medicina Intensiva Brasileira, 2006
RESULTADOS: Duzentos e trinta e sete médicos responderam os formulários. Em todos os três casos foram observadas escolhas de intervenção terapêutica completamente distintas, nenhuma delas obtendo mais de $80 \%$ de concordância. Quando se comparam as escolhas direcionadas pelos resultados da ecocardiografia, observou-se a persistência da variação de escolhas e que nenhuma delas alcançou número suficiente para ser considerada homogênea.

CONCLUSÕES: Semelhantemente ao estudo original, observou-se total heterogeneidade nas condutas dirigidas pelo CAP, o que, em última instância, pode indicar conhecimento inadequado de conceitos fisiopatológicos básicos, e que o ensino nos cursos médicos precisa ser revisto e aprimorado.

Unitermos: Cateter de Artéria Pulmonar, Ecocardiografia, Evolução, Monitorização Hemodinâmica, Tomando Decisões

\section{SUMMARY}

BACKGROUND AND OBJECTIVES: Use of Pulmonary Artery Catheter (PAC) is still a debatable issue, mainly due to questions raised about its security and efficacy. This study reproduced in a sample of Brazilian physicians, another one conducted amidst American doctors, in which was pointed out the heterogeneity of clinical decisions guided by data obtained from PAC.

METHODS: During the Brazilian Congress of Intensive Care Medicine (Curitiba 2004), doctors were asked to answer a survey form with three vignettes. Each of them contained PAC data and one half of the surveys contained echocardiographic information. Every doctor was asked to select one of six interventions for each vignette. A homogeneous answer was considered when it was selected by at least $80 \%$ of the respondents.

RESULTS: Two hundred and thirty seven doctors answered the questionnaires. They selected completely different therapeutic interventions in all three vignet- 
tes and none of the interventions achieved more than $80 \%$ agreement. Variability persisted with the choices guided by echocardiography.

CONCLUSIONS: As in the original study, we observed total heterogeneity of therapeutic interventions guided by CAP and echocardiography. These results could be caused by lack of knowledge about basic pathophysiologic concepts and maybe we had to improve its teaching at the medical school benches.

Key Words: Decision Making, Echocardiography, Hemodynamic Monitoring, Outcome, Pulmonary Artery Catheter

\section{INTRODUÇÃO}

O desenvolvimento do cateter de artéria pulmonar (CAP) na década de $1970^{1}$ permitiu melhor entendimento fisiopatológico dos estados de choque ${ }^{2,3}$ propiciando um tratamento dirigido ao distúrbio circulatório. Posteriormente, evidenciou-se que a avaliação do estado hemodinâmico com base em parâmetros clínicos nem sempre é fidedigna ${ }^{4-7}$ e que muitas vezes pode levar a condutas equivocadas, o que certamente afeta o desfecho do paciente crítico.

Embora seja um instrumento desenvolvido para identificar o perfil hemodinâmico, com freqüência o CAP é confundido com medida terapêutica. As conclusões de estudos feitos para avaliar a sua eficácia em pacientes críticos mostraram inúmeras divergências, com alguns autores apontando para aumento de mortalidade com o seu emprego ${ }^{8,9}$ e outros não demonstrando efeito sobre a mortalidade com o uso do CAP ${ }^{10-13}$.

Provavelmente, a falta de uniformidade nos resultados dos estudos com CAP se devem a vários fatores, como conhecimento insuficiente para utilizar esta tecnologia ${ }^{13,14}$, uso da monitorização hemodinâmica após a instalação de disfunções orgânicas ${ }^{8,15}$, uso equivocado de medidas terapêuticas ${ }^{16}$ e até a falta de protocolos de tratamento com objetivos terapêuticos definidos.

A variabilidade nas intervenções de pacientes sob monitorização com CAP foi estudada recentemente. Manu Jain e col. ${ }^{17}$ publicaram um estudo transversal nos quais avaliaram, através de questionário, a uniformidade de condutas de especialistas em Medicina Intensiva, membros do American College of Chest Physicians e da Society of Critical Care Medicine. Os autores encontraram uma grande variabilidade nas condutas selecionadas pelos intensivistas, o que os levou a concluir que ao se avaliar a eficiência do CAP, protocolos rígidos de conduta deveriam ser utilizados.
Como no nosso meio há falta de dados sobre o uso do CAP de uma maneira geral e este tema em particular, esse estudo foi reproduzido durante o Congresso Brasileiro de Medicina Intensiva (Curitiba, 2004), entre médicos especialistas brasileiros.

\section{MÉTODO}

Após validação do questionário para a língua portuguesa por três médicos, os participantes do congresso foram convidados a respondê-lo. Dentro da bolsa de cada participante do congresso foi deixado um questionário. O questionário foi baseado em três cenários clínicos brevemente descritos (Anexo 1). Cada caso possuía dados obtidos com o CAP e metade dos questionários continha, ainda, dados obtidos por ecocardiografia transtorácica. Foi solicitado que, em cada caso clínico, o médico fizesse a escolha de uma intervenção entre seis opções. Na seção inicial do formulário foram solicitados os dados demográficos sobre sua experiência com o método. Na segunda seção foram colocados os casos clínicos com os respectivos resultados de monitorização hemodinâmica. Cada caso continha informações a respeito da história clínica, exame físico, gasometria arterial, parâmetros de ventilação e mecânica respiratória, interpretação da radiografia de tórax e resultados detalhados obtidos com o cateter de artéria pulmonar. Além disso, 50\% dos questionários incluíam informações de exame ecocardiográfico transtorácico. Após cada descrição de caso, os médicos foram solicitados a especificar se havia indicação para a passagem do CAP e a escolher uma única de seis opções (cinco intervenções específicas comuns: infusão de solução fisiológica, dobutamina, dopamina, nitroprussiato, furosemida e uma sexta opção, com nenhuma das respostas). Como no trabalho original, foi assumido que uma resposta homogênea resultaria em escolha selecionada por pelo menos $80 \%$ dos respondedores. $\mathrm{O}$ teste Exato de Fisher e o Qui-quadrado foram usados para comparar a distribuição das escolhas entre os respondedores, com e sem ecocardiografia transtorácica e entre os diferentes grupos demográficos. Estatística significativa foi definida como $p<0,05$.

\section{RESULTADOS}

Duzentos e trinta e sete médicos atenderam à solicitação. O tempo médio de formado era de 13,3 \pm 8,3 anos. Cento e trinta e dois $(55,7 \%)$ eram especialistas em Medicina Intensiva, 58 (24,5\%) não eram titulados 
pela AMIB e $47(19,8 \%)$ eram médicos residentes. A maioria dos médicos (114 ou 48,1\%) havia instalado de um a cinco cateteres no mês prévio ao evento, 32 $(13,5 \%)$ tinham instalado mais de seis cateteres e 91 $(38,4 \%)$ não haviam realizado procedimentos com CAP. Cento e vinte e nove $(54,4 \%)$ dispunham de informações do ecocardiograma, enquanto 108 (45,6\%) receberam apenas os resultados obtidos através do CAP. No caso 1 não houve consenso a respeito da indicação do CAP e 21,5\% não concordaram com a necessidade de instalá-lo. Nos casos 2 e 3 a indicação do CAP foi consensual para $85,2 \%$ e $84,8 \%$, respectivamente. Em todos os três casos foram observadas escolhas de intervenção terapêutica completamente distintas, nenhuma delas obtendo mais de $80 \%$ de concordância, como se pode observar na figura 1 . No caso 1 , a escolha mais freqüente foi a administração de fluidos, com $35 \%$ das respostas; no caso 2 também a administração de fluidos, com $42 \%$; e no caso 3 , dobutamina foi a escolha preferencial de $38 \%$ dos médicos. Quando são comparadas as escolhas orientadas pelos resultados da ecocardiografia, observa-se a persistência da variação de escolhas, sendo que nenhuma delas alcançou número suficiente para ser considerada homogênea (Tabela 1).

A principal escolha terapêutica dos médicos com título de especialista no $1^{\circ}$ caso, foi por fluidos (48\% ou $36,4 \%)$, assim como no $2^{\circ}$ caso $(65 \%$ ou $49,2 \%)$ e por dobutamina no $3^{\circ}$ caso $(61 \%$ ou $46,2 \%)$. Sem o ECO, a principal escolha dos médicos titulados foi por fluidos no $1^{\circ}(15 \%$ ou $30 \%)$ e $2^{\circ}$ caso $(20 \%$ ou $40 \%)$ e por dobutamina (25\% ou $50 \%$ ) no $3^{\circ}$ caso. Quando se disponibilizou o ECO, as escolhas permaneceram erráticas para este grupo, com $33(40,1 \%)$ e $45(54,9 \%)$ optando por fluidos no $1^{\circ}$ e $2^{\circ}$ casos, respectivamente, e $36(43,9 \%)$ por dobutamina no $3^{\circ}$ caso. Entre os mé- dicos não titulados ou residentes, as escolhas foram ainda mais aleatórias e nenhuma das opções terapêuticas alcançou mais do que $35 \%$ em qualquer dos três casos. Em relação ao tempo de formado, a primeira escolha foi por fluidos $(35,4 \%$ para os com menos de dez anos e $34,5 \%$ para os com mais de 10 anos de formado) no primeiro e segundo caso (33,3\% e 48,2\%) e dobutamina (40,6\% versus $36 \%)$ no terceiro.

Um panorama das intervenções escolhidas está apresentado tabelas 1 e 2 .

Tabela 1 - Escolha de Intervenções com e sem Ecocardiograma

\begin{tabular}{|c|c|c|c|}
\hline \multicolumn{4}{|c|}{ Caso 1} \\
\hline & Todos (\%) & Eco (\%) & Sem Eco (\%) \\
\hline $\mathrm{NaCl}$ a $0,9 \%$ & $83(35,0)$ & $48(37,2)$ & $35(32,4)$ \\
\hline Dobutamina & $56(23,6)$ & $34(26,3)$ & $22(20,4)$ \\
\hline Dopamina & $17(7,2)$ & $10(7,8)$ & $7(6,5)$ \\
\hline Nitroprussiato & $15(6,3)$ & $8(6,2)$ & $7(6,5)$ \\
\hline Furosemida & $23(9,7)$ & $8(6,2)$ & $15(13,9)$ \\
\hline Nenhuma & $43(18,2)$ & $21(16,3)$ & $22(20,4)$ \\
\hline \multicolumn{4}{|c|}{ Caso 2} \\
\hline & Todos (\%) & Eco (\%) & Sem Eco (\%) \\
\hline $\mathrm{NaCl}$ a $0,9 \%$ & $100(42,2)$ & $61(47,3)$ & $39(36,1)$ \\
\hline Dobutamina & $10(4,2)$ & $2(1,5)$ & $8(7,4)$ \\
\hline Dopamina & $75(31,6)$ & $39(30,2)$ & $36(33,3)$ \\
\hline Nitroprussiato & $6(2,5)$ & $3(2,3)$ & $3(2,8)$ \\
\hline Furosemida & $8(3,4)$ & $4(3,1)$ & $4(3,7)$ \\
\hline Nenhuma & $38(16,1)$ & $20(15,5)$ & $18(16,7)$ \\
\hline \multicolumn{4}{|c|}{ Caso 3} \\
\hline & Todos (\%) & Eco (\%) & Sem Eco (\%) \\
\hline $\mathrm{NaCl}$ a $0,9 \%$ & $48(20,3)$ & $30(13,1)$ & $18(16,6)$ \\
\hline Dobutamina & $90(38,0)$ & $45(34,9)$ & $45(41,6)$ \\
\hline Dopamina & $27(11,4)$ & $14(10,8)$ & $13(12,0)$ \\
\hline Nitroprussiato & $7(2,9)$ & $3(2,3)$ & $4(3,7)$ \\
\hline Furosemida & $6(2,5)$ & $4(3,1)$ & $3(2,8)$ \\
\hline Nenhuma & $59(24,9)$ & $34(26,3)$ & $25(23,1)$ \\
\hline
\end{tabular}

Nota: Nenhuma intervenção foi escolhida por pelo menos $80 \%$ dos participantes nos três casos e independente das informações adicionais do ecocardiograma.

Figura 1 - Variabilidade nas Intervenções nos Três Casos Apresentados

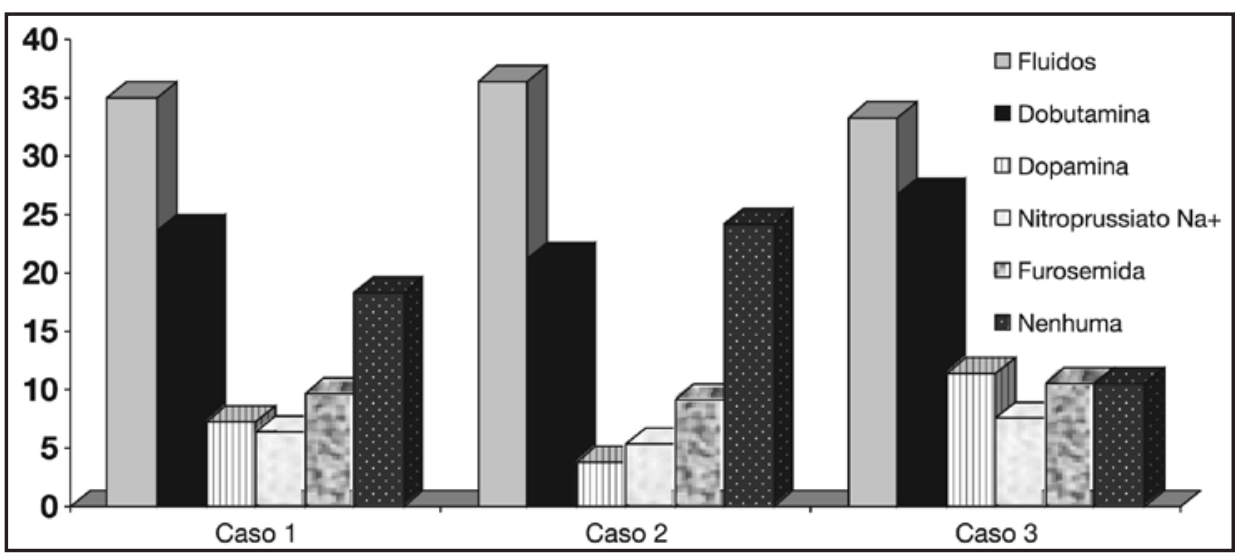


MENDES, REZENDE, DIAS E COL.

Tabela 2 - Comparação entre Especialistas em Medicina Intensiva e Médicos sem Título de Especialista ou Residentes.

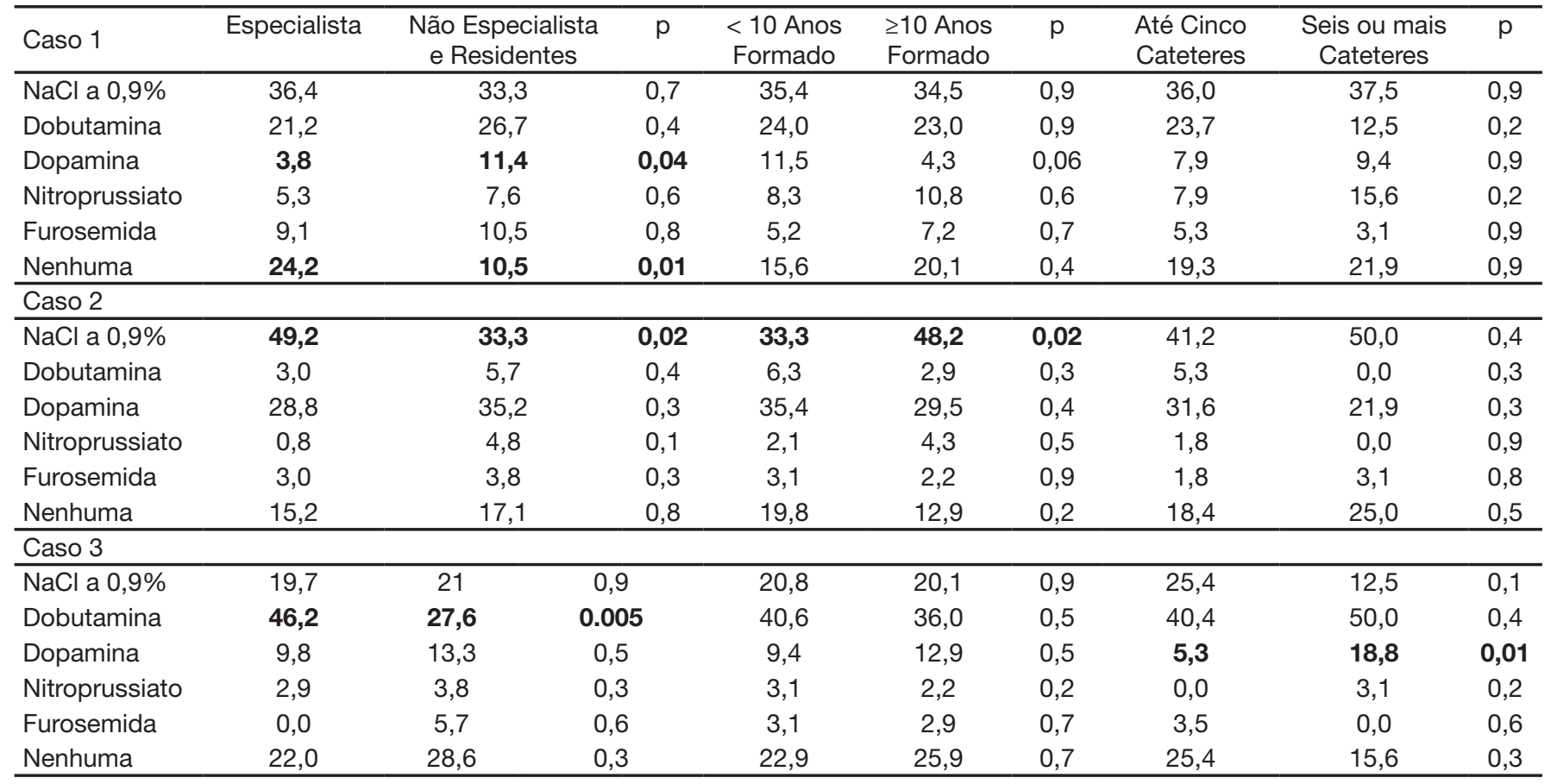

Valores expressos em porcentagem

\section{DISCUSSÃo}

Apesar de há mais de três décadas o CAP proporcionar uma abordagem fisiológica nos estados de choque e instabilidade hemodinâmica, ainda discute-se sua efetividade e segurança ${ }^{18,19}$.

Os resultados de estudos que utilizaram o CAP são conflitantes, com alguns mostrando benefício ${ }^{20-22}$ e outros demonstrando não haver vantagens na utilização das informações obtidas com o emprego dessa tecnologia em pacientes críticos ${ }^{8,9,23-25}$. Essa discrepância deve-se em grande parte aos variados métodos aplicados para avaliação da eficácia do cateter, às diferentes populações estudadas, à falta de protocolos rígidos guiados por dados obtidos pela monitorização com o CAP, à instalação do cateter em momentos diferentes na evolução dos processos fisiopatológicos, entre outros. De todos os argumentos discutidos, talvez o mais consistente seja o fato de o CAP ser apenas um instrumento de monitorização. Portanto, ele deve ser eficaz em guiar a escolha da intervenção a ser feita ${ }^{6,7,20-22}$, não tendo impacto direto sobre a evolução do paciente.

Um outro importante aspecto nessa equação seria a possível limitação dos profissionais envolvidos com a utilização do cateter em interpretar e implementar terapias consistentes guiadas pelos dados obtidos.
Iberti e col..$^{13}$ demonstraram uma grande variação de interpretações e compreensão das informações obtidas com o CAP entre profissionais médicos de UTI nos Estados Unidos e Canadá. Posteriormente, Gnaegi e col. ${ }^{14}$ utilizaram-se do mesmo questionário de lberti e constataram que o conhecimento sobre o CAP não era uniformemente satisfatório.

O estudo de Jain e col. ${ }^{17}$ e o presente estudo, baseados no mesmo método de pesquisa apresentaram similaridades, principalmente em relação à heterogeneidade das intervenções escolhidas, o que reflete, em última instância, variabilidade na interpretação da fisiopatologia dos casos apresentados. Embora o número de questionários avaliados no presente estudo tenha sido aproximadamente o dobro daqueles no estudo de Jain e col. ${ }^{17}$ houve a mesma tendência à variabilidade.

Outras particularidades de ambos os estudos merecem ser ressaltada: enquanto no estudo americano o número de médicos que declararam não ter prática com o cateter foi de 35 (9\%), no brasileiro, apesar de terem declarado familiaridade com o método, 91 (38,4\%) não haviam realizado procedimentos com o CAP no mês precedente ao preenchimento do questionário, o que pode refletir desde uma atitude mais conservadora na abordagem do paciente com instabilidade hemodinâmica até a não disponibilidade de recursos. No estudo original, todos 
os médicos respondedores tinham titulação de especialista reconhecida pela American College of Chest Physician (ACCP) e Society of Critical Care Medicine (SCCM), enquanto que no presente estudo, aproximadamente a metade (132 ou 55,7\%) era de especialistas em Medicina Intensiva. O restante era composto de médicos não titulados (58 ou $24,5 \%$ ) ou de médicos residentes (47 ou $19,8 \%)$. Um viés que merece ser considerado, e que é comum a toda pesquisa espontânea, é a possibilidade de que os médicos que se disponibilizaram a respondê-la pudessem ter maior simpatia pelo método e, conseqüentemente, maior familiaridade. Seja isso verdade, mais preocupantes são os resultados.

A indicação do CAP, no trabalho original, foi postulada por $69 \%$ dos médicos, enquanto que neste estudo a proporção de indicações foi maior, apesar de no caso 1 não ter havido consenso e $21,5 \%$ não terem concordado com essa necessidade. Nos casos 2 e 3 a indicação do CAP foi consensual para $85,2 \%$ e $84,8 \%$, respectivamente.

Este estudo e o trabalho de Jain e col. ${ }^{17}$ agregaram resultados obtidos pela ecocardiografia aos do CAP. Este elemento, além de atribuir certa particularidade aos trabalhos, permitem outros questionamentos interessantes. Alguns estudos já demonstraram que dados obtidos pelo CAP são reprodutíveis pelo ecocardiograma $(E C O)^{26}$ e aquele é considerado como "padrão ouro" na validação deste, para monitoração hemodinâmica. Seria esperado que dados do ECO associados aos do CAP apenas reforçassem uma tendência na escolha de condutas. Em ambos os estudos, no primeiro caso clínico, os dados do ECO aumentaram a heterogeneidade na tomada de decisão e em particular no segundo e terceiro caso, a modificaram. Se for feito uma análise, as escolhas terapêuticas feitas em cada caso, chamam atenção algumas peculiaridades. No segundo caso, um paciente com choque séptico desenvolve hipotensão. As opções que poderiam ser julgadas mais apropriadas seriam a reposição de fluidos e administração de vasopressores. O ECO mostrou que o paciente possuiu significativa hipertrofia de ventrículo esquerdo, inclusive com gradiente intraventricular. Estes dados sugerem fortemente a administração de fluidos e fundamentalmente contra-indicam o uso de fármacos adrenérgicos. Ainda assim, 30\% dos médicos no estudo original e $31,6 \%$ no presente estudo, que tiveram acesso ao ECO, propuseram uso de dopamina. No terceiro caso, em ambos os estudos, podem-se notar que independentemente da alternativa mais adequada, houve decisões totalmente opostas como uso de vasodilatadores e vasopressores. Estes resultados reforçam ainda mais a hipótese de falta de uniformidade nas condutas, representada por interpretação inadequada das variáveis hemodinâmicas obtidas através do CAP.

Ambos os trabalhos propuzeram avaliar exclusivamente a uniformidade de tomada de conduta por médicos intensivistas baseados em dados do CAP. O que foi verificado no trabalho original e reproduzido quase que integralmente neste estudo foi uma heterogeneidade significativa na conduta tomada pelos médicos. Esses resultados reforçam a idéia de que os resultados conflitantes evidenciados pelos estudos sobre o CAP podem ser explicados não pelo potencial malefício do cateter, mas por intervenções terapêuticas diferentes e possivelmente inadequadas. Outro fator a ser considerado é que houve uma tendência a empregar métodos invasivos em pacientes nos quais o curso clínico é desfavorável. Havendo disfunção de três sistemas após cinco ou seis dias, a mortalidade aproxima-se de $100 \%$, não havendo maneira de se saber se essa tendência seria alterada pelo CAP ${ }^{27}$.

O uso apropriado do CAP, no entanto, depende do cumprimento de várias etapas, além da vigilância constante ao paciente. Esses resultados sugerem que exista ainda uma generalizada e inadequada interpretação dos dados obtidos pelo CAP e tomadas de decisão também conseqüentemente impróprias. Ou seja, diante do mesmo quadro clínico, as condutas são arbitrárias e díspares.

Um aspecto de importância a ser destacado é que as escolhas dos médicos brasileiros foram mais coincidentes com o que Jain e col. ${ }^{17}$ que consideraram como resposta mais adequada para cada um dos três casos: volume, volume e dobutamina, enquanto que no estudo original, tal fato não ocorreu.

\section{CONCLUSÃO}

Talvez a discussão em torno do CAP seja mais de ordem fisiopatológica do que do próprio método em si. O cerne da questão talvez repouse em um conhecimento inadequado de conceitos fisiopatológicos básicos, cujo ensino nos cursos médicos precise ser revisto e aprimorado.

O presente estudo reproduziu quase que integralmente os resultados do estudo original e com raros e insignificantes desvios, os autores observaram uma total heterogeneidade nas condutas tomadas com base nas informações fornecidas e infelizmente corroboraram tais resultados também em nosso meio. 
Anexo 1 - Casos Clínicos com Informações do Cateter de Artéria Pulmonar e Ecocardiograma

\begin{tabular}{|c|c|c|}
\hline Jaso Clínico 1 & Clínico 2 & aso CIInIC \\
\hline 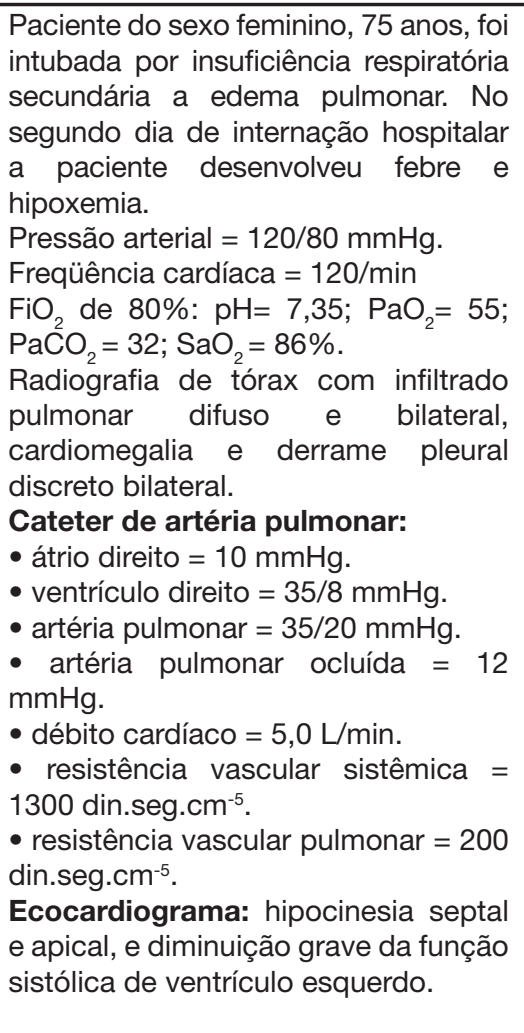 & 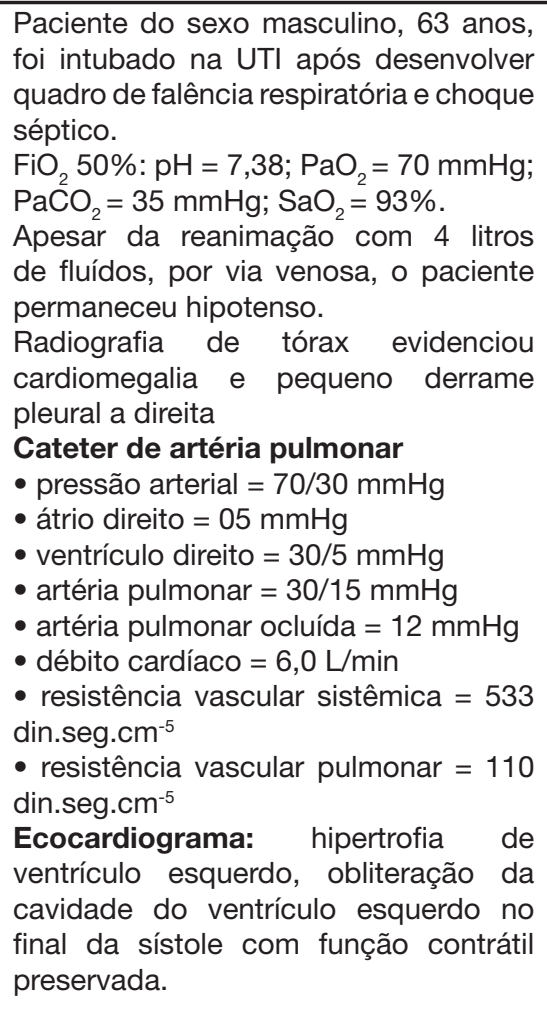 & 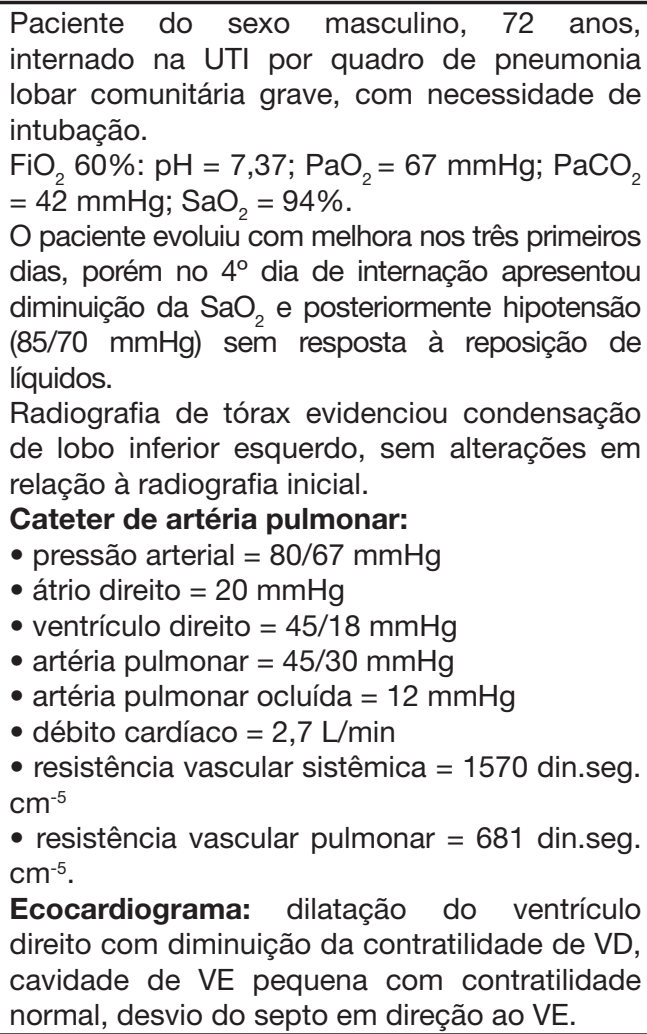 \\
\hline
\end{tabular}

\section{REFERÊNCIAS}

01. Swan HJ, Ganz W, Forrester $\mathrm{J}$ et al - Catheterization of the heart in man with use of a flow-directed balloon-tipped catheter. N Engl J Med, 1970;283:447-451

02. Forrester JS, Diamond G, Chatterjee K et al - Medical therapy of acute myocardial infarction by application of hemodynamic subsets (second of two parts). N Engl J Med, 1976;295:1404-1413.

03. Parker MM, Shelhamer JH, Bacharach SL et al - Profound but reversible myocardial depression in patients with septic shock. Ann Intern Med, 1984; 100:483-490.

04. Connors AF Jr, McCaffree DR, Gray BA - Evaluation of right-heart catheterization in the critically ill patient without acute myocardial infarction. $\mathrm{N}$ Eng J Med, 1983;308:263-267.

05. Steingrub JS, Celoria G, Vickers-Lahti $M$ et al - Therapeutic impact of pulmonary artery catheterization in a medical/surgical ICU. Chest, 1991;99:1451-1455.

06. Mimoz O, Rauss A, Rekik N et al - Pulmonary artery catheterization in critically ill patients: a prospective analysis of outcome changes associated with catheter-prompted changes in therapy. Crit Care Med, 1994;22:573-579.

07. Coles NA, Hibberd M, Russell $\mathrm{M}$ et al - Potential impact of pulmonary arter catheter placement on short-term management decisions in the medical intensive care unit. Am Heart J, 1993;126:815-819.

08. Connors AF Jr, Speroff T, Dawson NV et al - The effectiveness of right heart catheterization in the initial care of critically ill patients. SUPPORT Investigators. JAMA, 1996;276:889-897.

09. Cohen MG, Kelly RV, Kong DF et al - Pulmonary artery catheterization in acute coronary syndromes: insights from the GUSTO Ilb and GUSTO III trials. Am J Med, 2005;118:482-488.

10. Afessa B, Spencer S, Khan W et al - Association of pulmonary artery catheter use with in-hospital mortality. Crit Care Med, 2001;29:1145-1148.

11. Yu DT, Platt R, Lanken PN et al - Relationship of pulmonary artery catheter use to mortality and resource utilization in patients with severe sepsis. Crit Care Med, 2003;31:2734-2741.

12. Harvey $S$, Harrison DA, Singer $M$ et al - Assessment of the clinical effectiveness of pulmonary artery catheters in management of patients in intensive care (PAC-Man): a randomised controlled trial. Lancet, 2005;366:(9484):472-477.
13. Iberti TJ, Fischer EP, Leibowitz AB et al - A multicenter study of physicians' knowledge of the pulmonary artery catheter. Pulmonary Artery Catheter Study Group. JAMA, 1990;264:2928-2932.

14. Gnaegi A, Feihl F, Perret C - Intensive care physicians' insufficient knowledge of right-heart catheterization at the bedside: time to act? Crit Care Med, 1997;25:213-220.

15. Kern JW, Shoemaker WC - Meta-analysis of hemodynamic optimization in high-risk patients. Crit Care Med, 2002;30:1686-1692.

16. Hayes MA, Timmins AC, Yau EH et al - Elevation of systemic oxygen delivery in the treatment of critically ill patients. N Engl J Med, 1994;330:1717-1722.

17. Jain $M$, Canham $M$, Upadhyay $D$ et al - Variability in interventions with pulmonary artery catheter data. Intensive Care Med, 2003;29:2059-2062.

18. De Backer D - Hemodynamic assessment: the technique or the physician at fault? Intensive Care Med, 2003;29:1865-1867.

19. Dalen JE - PA catheter-guided therapy does not benefit critically ill patients. Am J Med, 2005:118:449-451.

20. Shoemaker WC, Appel PL, Kram HB et al - Prospective trial of supranorma values of survivors as therapeutic goals in high-risk surgical patients. Chest, 1988;94:1176-1186.

21. Boyd O, Grounds RM, Bennett ED - A randomized clinical trial of the effect of deliberate perioperative increase of oxygen delivery on mortality in highrisk surgical patients. JAMA, 1993;270:2699-2707.

22. Wilson J, Woods I, Fawcett $\mathrm{J}$ et al - Reducing the risk of major elective surgery: randomised controlled trial of preoperative optimisation of oxygen delivery. BMJ, 1999:318:1099-1103.

23. Gattinoni L, Brazzi L, Pelosi P et al - A trial of goal-oriented hemodynamic therapy in critically ill patients. SvO2 Collaborative Group. N Engl J Med, 1995;333:1025-1032.

24. Alia I, Esteban A, Gordo F et al - A randomized and controlled trial of the effect of treatment aimed at maximizing oxygen delivery in patients with severe sepsis or septic shock, Chest, 1999;115:453-461.

25. Richard C, Warszawski J, Anguel N et al - Early use of the pulmonary artery catheter and outcomes in patients with shock and acute respiratory distress syndrome: a randomized controlled trial. JAMA, 2003;290:2713-2720.

26. Brown JM - Use of echocardiography for hemodynamic monitoring. Crit Care Med, 2002;30:1361-1364.

27. Weil MH - The assault on the Swan-Ganz catheter: a case history of constrained technology, constrained bedside clinicians, and constrained monetary expenditures. Chest, 1998;113:1379-1386 